\title{
Precise Molecular Engineering of Type I Photosensitizers with Near-Infrared Aggregation-Induced Emission for Image-Guided Photodynamic Killing of Multidrug-Resistant Bacteria
}

\author{
Peihong Xiao, ${ }^{[a, b, c]}$ Zipeng Shen, ${ }^{[a]}$ Deliang Wang, ${ }^{[a, b]}$ Yinzhen Pan, ${ }^{[a]}$ Ying Li, ${ }^{*[a]}$ Junyi Gong, ${ }^{[c]}$ Lei \\ Wang, ${ }^{[a]}$ Dong Wang ${ }^{*[a]}$ and Ben Zhong Tang ${ }^{*[, d]}$
}

[a] Dr. P. Xiao, Z. Shen, Dr. D. Wang, Y. Pan, Prof. Y. Li, Prof. L. Wang, Prof. D. Wang

Center for AIE Research, Shenzhen Key Laboratory of Polymer Science and Technology, Guangdong Research Center for Interfacial Engineering of

Functional Materials, College of Materials Science and Engineering

Shenzhen University

Shenzhen 518060, China

E-mail: wangd@szu.edu.cn

[b] Dr. P. Xiao, Dr. D. Wang, Prof. L. Wang

Key Laboratory of Optoelectronic Devices and Systems of Ministry of Education and Guangdong Province, College of Physics and Optoelectronic

Engineering

Shenzhen University

Shenzhen 518060, China

[c] Dr. P. Xiao, Dr. J. Gong, Prof. B. Z. Tang

Department of Chemistry, Hong Kong Branch of Chinese National Engineering Research, Center for Tissue Restoration and Reconstruction

The Hong Kong University of Science and Technology

Clear Water Bay, Kowloon, Hong Kong 999077, China

E-mail: tangbenz@ust.hk

[d] Prof. B. Z. Tang

Shenzhen Institute of Molecular Aggregate Science and Engineering, School of Science and Engineering

The Chinese University of Hong Kong

Shenzhen, 2001 Longxiang Boulevard, Longgang District, Shenzhen City, Guangdong 518172, China

E-mail: tangbenz@cuhk.edu.cn

\begin{abstract}
Multidrug resistance (MDR) bacteria pose a serious threat to human health. The development of alternative treatment modalities and therapeutic agents for treating MDR bacteria-caused infections remains a global challenge. Herein, we rationally designed and successfully developed a series of near-infrared (NIR) anion- $\pi^{+}$ photosensitizers featuring aggregation-induced emission (AIE-PSs) for broad-spectrum MDR bacteria eradication. Due to the strong intramolecular charge transfer (ICT) and enhanced highly efficient intersystem crossing (ISC), these electron-rich anion- $\pi^{+}$AIE-PSs showed boosted type I reactive oxygen species (ROS) generation capability involving hydroxyl radicals and superoxide anion radicals, and up to $99 \%$ photodynamic killing efficacy was achieved for both Methicillin-resistant Staphylococcus aureus (MRSA) and multi-drug resistant Escherichia coli (MDR E.coli) under a low dose white light irradiation (16 $\left.\mathrm{mW} \mathrm{cm}^{-2}\right)$. In vivo experiments confirmed that one of these AIE-PSs exhibited excellent therapeutic performance in curing MRSA or MDR E.coli-infected wounds with negligible side-effects. The study would thus provide useful guidance for the rational design of high-performance type I AIE-PSs to overcome antibiotic resistance.
\end{abstract}

\section{Introduction}

Pathogenic bacterial infections cause severe diseases and rising mortality, which poses a serious threat to global public health. ${ }^{[1]}$ Since the discovery of penicillin for the treatment of bacterial infections in 1928, antibiotics have brought dawn for humans to combat bacteria. ${ }^{[2,3]}$ However, due to the widespread clinical abuse of antibiotics in the past decades, the emergence of multidrug resistant (MDR) bacteria may bring us back to the "prehistoric era" if we do not have effective antibiotics. ${ }^{[4-6]}$ Given the circumstance, continuous exploration of new antibiotics is highly desirable, however, involves time-consuming and costly manipulations. Worse still, the development of new antibiotics usually can not keep up with the evolution of MDR bacteria, leading to increasingly serious antibiotic resistance..$^{[7,8]}$ According to the prediction of the Interagency Coordination Group on Antimicrobial Resistance (IACG), there are at least 700,000 deaths caused by resistant infections every year in the world. If no action is taken, the number will increase to annual 10 million by $2050 .^{[9]}$ Evidently, exploiting alternative treatment modalities and therapeutic agents against MDR bacteria are urgently needed.

Photodynamic therapy (PDT), with advantages combination of noninvasiveness, specific spatiotemporal selectivity and limited drug resistance, has been recognized as an effective and alternative stragety for infection treatment. ${ }^{[10-13]}$ The performance of PDT heavily relies on photosensitizers (PSs) that generate reactive oxygen species (ROS) to inactivate bacteria upon light irradiation. ${ }^{[14-17]}$ Generally, ROS can be divided into two categories, (1) Type I ones including $\cdot \mathrm{OH}, \mathrm{O}_{2}^{-\cdot}$, and $\mathrm{H}_{2} \mathrm{O}_{2}$ produced by electron transfer procedure, and (2) Type II one that is ${ }^{1} \mathrm{O}_{2}$ enerated by energy exchange process. ${ }^{[18,19]}$ Compared to the noticeable successes of PDT in the clinical treatment towards cancer, the full promise of antimicrobial PDT, however, has not yet been achieved due to the lack of ideal PSs sharing excellent targeting ability and low side-effects. ${ }^{[20-24]}$ In addition, most traditional fluorescent PSs suffer from aggregation-caused quenching (ACQ), leading to undesired weak fluorescence intensity and poor ROS generation efficiency, which severely hampers the application of antimicrobial PDT in clinical practice. ${ }^{[25]}$ Fortunately, luminogens with aggregationinduced emission (AIEgens) offer a brand-new solution, which is a concept established by our research group in 2001. ${ }^{[26-28]}$ Completely different from the ACQ phenomenon, AIEgens show weak or no emission when dissolved in solutions but remarkably amplified emission in the aggregation state due to the restriction 
a

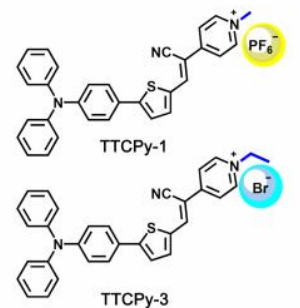

- High D-A structure with strong ICT characteristic

- Type I ROS generation capability

b
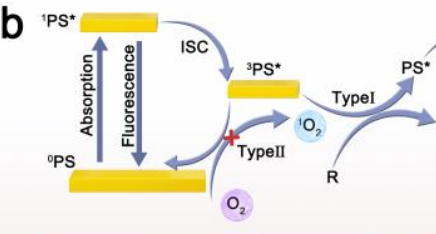

MDR Ecoli treat

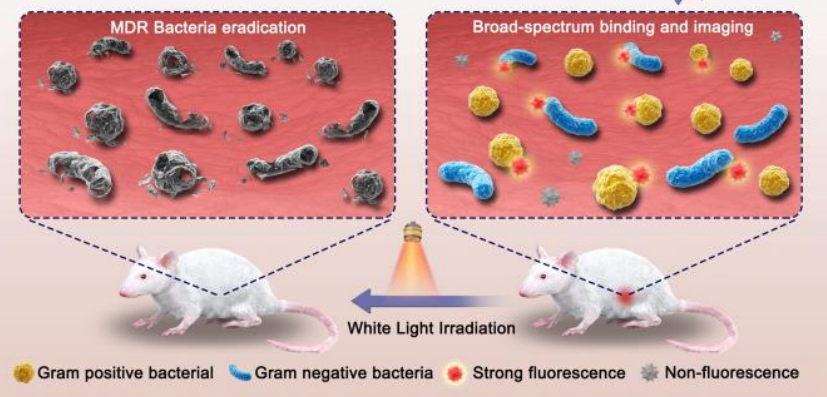

Figure 1. a) Molecular structures of designed type I AIE-PSs; b) Photophysical and photochemical mechanisms of type-I and type-II processes and imageguided broad spectrum antibacterial application of type I AIE-PSs.

of intramolecular motion (RIM). Although some AlEgenmediated antimicrobial PDT protocols have been developed in recent years, the following problems retain to be solved: 1) short absorption and emission wavelengths, which exhibit low tissue penetration and severe photo-damage to biosystem; 2) poor imaging ability for Gram negative $\left(\mathrm{G}^{-}\right)$bacteria, which comes from the insufficient restriction of intramolecular motion of AlEgens by the outer phospholipid membrane of the $\mathrm{G}^{-}$bacteria; 3) low eradication efficiency of drug-resistant bacteria, especially for MDR negative bacteria, which mainly due to the short lifetime and limited radius of ROS. ${ }^{[29-38]}$

In our previous work, a near infrared (NIR) AIEgen TTPy which could selectively image and eradicate of Gram-positive $\left(\mathrm{G}^{+}\right)$bacteria $S$. aureus was developed. ${ }^{[39]}$ This outcome was mainly resulted from the fact that the outer peptidoglycan layer of the $\mathrm{G}^{+}$bacteria can rapidly combine with the positively charged AIE molecules through electrostatic interaction, leading to the restriction of intramolecular motion and fluorescence turnon, while the outer phospholipid membrane of the $\mathrm{G}^{-}$bacteria did not have sufficient limitation on the molecular movement of AlEgens. ${ }^{[40]}$ Inspired by the application of nitrile groups in drug design to enhance binding affinity with target proteins through hydrogen bond interactions, covalent interactions, polar interactions, $\pi-\pi$ interactions and electrostatic interactions, ${ }^{[41,42]}$ we speculate that the introduction of a cyano group into TTPy could further restrict intramolecular motion by forming hydrogen bonds with the outer phospholipid membrane, thereby achieving lighting-up of both $\mathrm{G}^{-}$and $\mathrm{G}^{+}$bacteria. Besides, the enhanced donor-acceptor (D-A) strength could enormously promote intramolecular charge transfer (ICT), which could facilitate the

spatial separation of the highest occupied molecular orbital (HOMO) and the lowest unoccupied molecular orbital (LUMO), resulting in acceleration of intersystem crossing (ISC) process by minimizing energy level difference $\left(\Delta E_{\mathrm{st}}\right)$ between and singlet state $\left(S_{1}\right)$ and triplet state $\left(T_{1}\right)$. Furthermore, anion- $\pi^{+}$AlEgens possess an inherent advantage in providing electrons to excited PSs due to the electron-rich anionic reductant, which is rather critical in the design of free radical-type PSs. ${ }^{[43,44]}$ Therefore, nitrile-containing anion- $\pi^{+}$AIEgens fabricated with strong ICT characteristic will not only achieve broad spectrum imaging of bacteria, but also can eradicate MDR bacteria by generating more destructive type I ROS.

To verify these hypotheses, we rationally designed four anion- $\pi^{+}$AlEgens (TTCPy-1, TTCPy-2, TTCPy-3 and TTCPy-4) comprising triphenylamine (TPA) fragment (working as an AIEactive electron donor, $D$ ), a thiophene unit (serving as $D$ and $\pi$ bridge), a carbon-carbon double bond ( $\pi$ bridge), cyano and pyridinium moiety (acting as double electron acceptors, A). The D-A strength and ISC process can be precisely regulated by changing the substituents on the pyridine salt and selecting different anions as counterions. The introduction of heavy halide ions in TTCPy-3 $(\mathrm{Br})$ and TTCPy-4 $\left(\mathrm{I}^{-}\right)$can facilitate the effective ISC process due to the efficient enhancement of spinorbit coupling (SOC) caused by heavy atom effect. ${ }^{[45,46]}$ TTCPy-1, TTCPy-2, TTCPy-3 and TTCPy-4 could achieve not only $\mathrm{G}^{+}$ bacteria imaging but also $G^{-}$bacteria imaging, implying that nitrile-containing AIEgens indeed enhance the binding ability to phospholipid membrane of $\mathrm{G}^{-}$bacteria. Thanks to the strong ICT intensity and electron-rich condition contributed by anions, TTCPy series all exhibit highly efficient Type I ROS generation capability, offering excellent in vitro/vivo fluorescence imaging guided PDT killing efficiency toward MDR bacteria. We expect this presented design strategy can be applied to the development of more AIE-active type I PSs to combat bacterial infection.

\section{Results and Discussion}

Compounds TTCPy-1, TTCPy-2, TTCPy-3 and TTCPy-4 were synthesized through a few reaction steps. The synthetic routes of these four compounds are depicted in Scheme S1. The common intermediate TTCPy was facile obtained by two steps of Suzuki coupling and condensation reaction using 4-bromo- $N$, $\mathrm{N}$-diphenylaniline and (5-formylthiophen-2-yl) boronic acid as starting materials. The alkylation of pyridine in TTCPy using iodomethane and subsequent ion exchange in saturated $\mathrm{KPF}_{6}$ solution were successively conducted and achieved a moderate yield of $54 \%$ for TTCPy-1. TTCPy-2, TTCPy-3 and TTCPy-4 were obtained from the corresponding iodoethane and bromoethane in the same procedure with high yields ranging from $58 \%$ to $79 \%$ (Figure S1-S15).

With TTCPy series compounds in hands, their optical properties were further evaluated. Different anions have little effect on the maximum absorption peaks, as illustrated in Figure 2A. The maximum absorptions of TTCPy-1, TTCPy-2, TTCPy-3, TTCPy-4 in DMSO were located at 532, 532, 534, and $534 \mathrm{~nm}$ respectively. TTCPy-1 and TTCPy-2 with hexafluorophosphate $\left(\mathrm{PF}_{6}{ }^{-}\right)$had a maximum emission peak at $654 \mathrm{~nm}$ and $650 \mathrm{~nm}$ respectively (Figure $2 \mathrm{~B}$ ), while the maximum emission peak of 

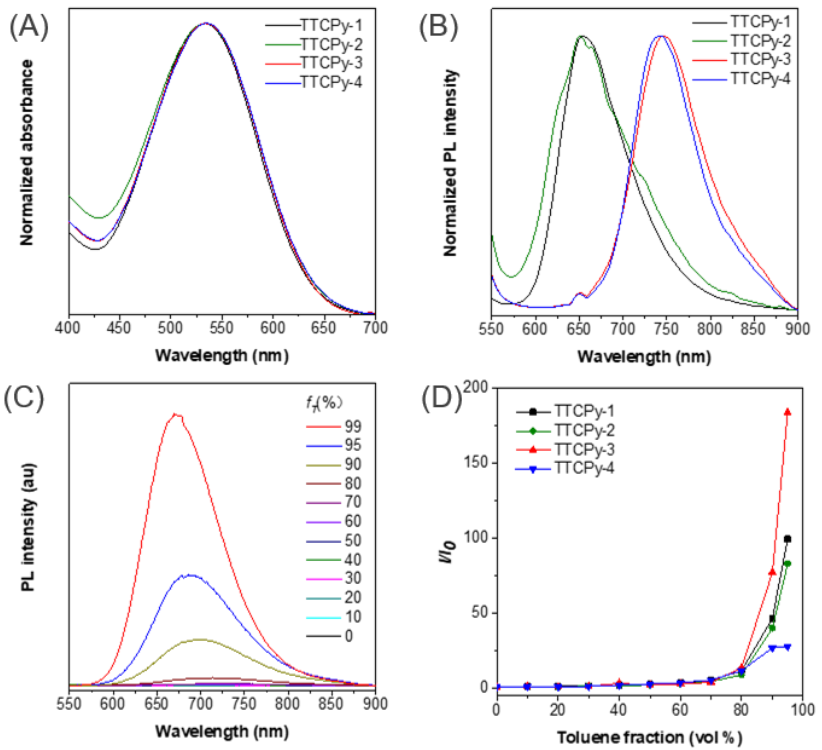

Figure 2. A) Normalized absorption spectra of TTCPy-1, TTCPy-2, TTCPy-3 and TTCPy-4 in the DMSO solution. B) Normalized PL spectra of TTCPy-1, TTCPy-2, TTCPy-3 and TTCPy-4 in the solid state. C) PL spectra of TTCPy-3 $\left(10 \times 10^{-6} \mathrm{M}\right)$ in DMSO/Toluene mixtures with different toluene fractions $\left(f_{T}\right)$. D) The plot of the relative emission intensity $\left(I / I_{0}\right)$ versus toluene fraction. $I_{0}$ and $I$ are the peak values of photoluminescence intensities of AIEgens $\left(10 \times 10^{-6} \mathrm{M}\right)$ in DMSO and DMSO/toluene mixtures, respectively.

TTCPy-3 and TTCPy-4 with bromide $\left(\mathrm{Br}^{-}\right)$and iodide $\left(\mathrm{I}^{-}\right)$as counterions red-shifted to $748 \mathrm{~nm}$ and $742 \mathrm{~nm}$ respectively, which may be ascribed to the anion- $\pi^{+}$interactions between the heavy halide ions and the positively charged pyridine rings. ${ }^{[47-49]}$ PL spectra of the TTCPy series were then measured in DMSO/toluene mixtures to evaluate their AIE tendency. As depicted in Figure 2C, 2D and Figure S16, when the toluene fraction $\left(f_{T}\right)$ was below $70 \%$, the $\mathrm{PL}$ intensities of these four compounds were quite weak, with negligible fluorescence quantum yields ranging from $0.5 \%$ to $0.9 \%$ in solution. Subsequently, increasing $f_{T}$ to $80 \%$, the PL intensities of TTCPy1, TTCPy-2, TTCPy-3 and TTCPy-4 amplified dramatically and reached to their maximum values at $99 \%$ of $f_{T}$, indicating their typical AIE features. Due to the twist intramolecular charge transfer (TICT) effect of TTCPy series, a slight blue shift in fluorescence emission was observed with the decrease in polarity of the solvent mixtures. It is noteworthy that TTCPy-3 with bromide $\left(\mathrm{Br}^{-}\right)$had the highest $\alpha_{\text {AIE }}$ value up to 184 , which may be attributed to much stronger anion $-\pi^{+}$interactions between the bromide anions and the positively charged pyridine ring that avoid $\pi-\pi$ stacking in the aggregation state. As shown in Table S1, the fluorescence quantum yields of TTCPy-1, TTCPy-2, TTCPy-3 and TTCPy-4 reached 12.9\%, 12.1\%, 19.2\% and $6.1 \%$ respectively in the aggregation state, which were about $100,83,184$ and 28 folds of those in DMSO solution $(0.9 \%, 0.8 \%, 0.5 \%$ and $0.7 \%$, respectively), further confirming their AIE characteristics.

The development of high-performance photosensitizers highly depends on the ISC rate and its ability in light absorption. [50] On one hand, ISC can be significantly amplified through HOMO-LUMO engineering to achieve a small energy gap between $S_{1}$ and $T_{1}$. On the other hand, ISC can be increased by incorporation of heavy atoms into their structures which can strengthen the spin-orbit coupling to boost the triplet quantum yields. It is generally accepted that electron-rich anion- $\pi^{+}$ AlEgens with strong ICT effect can generate more free radical ROS via type I mechanism. ${ }^{[43,44]}$ In addition, the suppressed molecular motions of AlEgens in the aggregation state are beneficial for ISC process, which is called as the aggregationinduced ISC. ${ }^{[51]}$ Therefore, as an elegant combination of all the above mentioned factors, TTCPy series are expected to be high performance AlE-active type I photosensitizers.

The total ROS-generating abilities of TTCPy-1, TTCPy-2 TTCPy-3 and TTCPy-4 in DMSO solution with 99\% PBS fractions were investigated using 2',7'-dichlorodihydrofluorescein $(\mathrm{DCFH})$ as an indicator under white light irradiation. As shown in Figure $3 \mathrm{~A}$, the fluorescence intensity of DCFH rapidly enhanced in the presence of these AIE-PSs along with the continuous irradiation due to the greatly suppressed nonradiative decay in the aggregation state, while negligible increase in fluorescence signal was detected for the irradiated solution with DCFH alone. Thanks to the strong heavy atom effect of bromine and iodine ions which can promote the ISC process, TTCPY- 3 and TTCPy- 4 had higher ROS generation ability than TTCPy-2 with an emission intensity nearly 550-fold
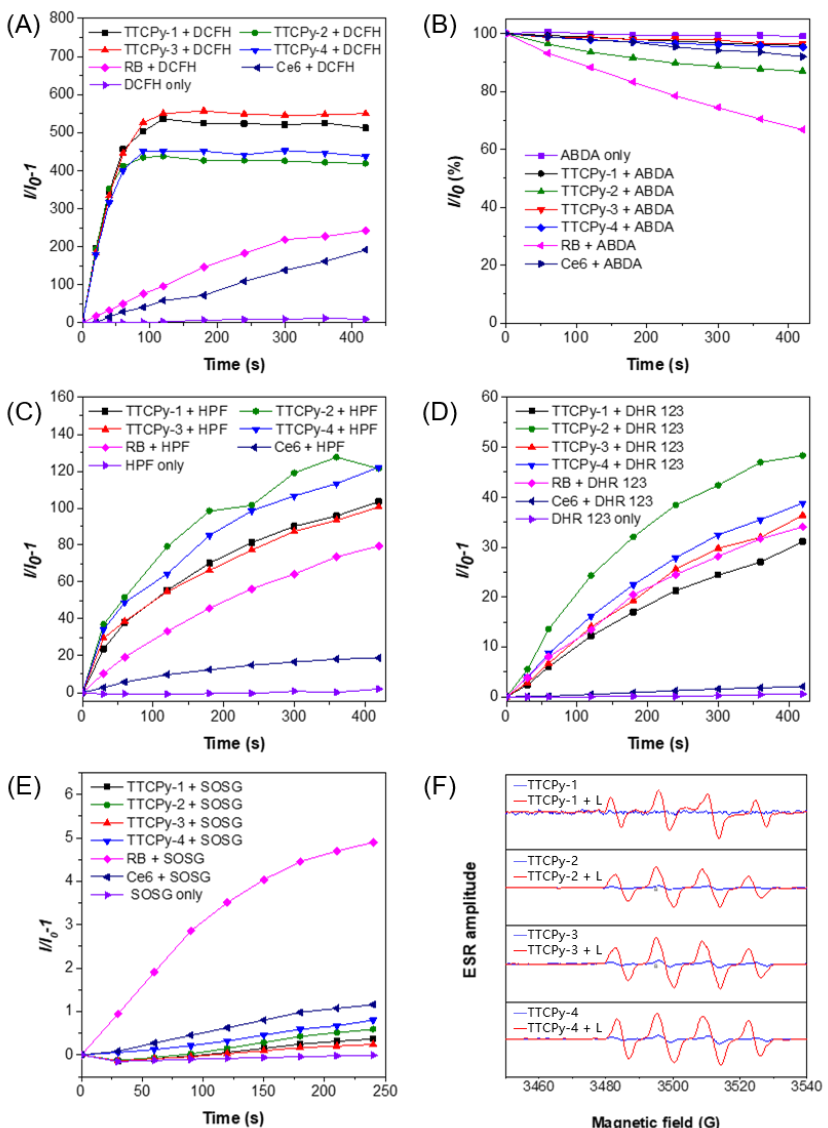

Figure 3. ROS generation of TTCPy-1, TTCPy-2, TTCPy-3 and TTCPy-4 upon white light irradiation. Relative changes in PL intensity of (A) DCFH (for overall ROS detection), (B) decomposition rates of $A B D A$ (for ${ }^{1} \mathrm{O}_{2}$ detection), (C) HPF (for $\bullet \mathrm{OH}$ detection), (D) DHR 123 (for $\mathrm{O}_{2}^{-\cdot}$ detection) and (E) SOSG (for ${ }^{1} \mathrm{O}_{2}$ detection) in the presence of TTCPy-1, TTCPy-2, TTCPy-3 or TTCPy$4(1 \mu \mathrm{M})$ upon white light irradiation $\left(16 \mathrm{~mW} \mathrm{~cm}^{-2}\right)$ for different time. $(F)$ ESR signals of DMPO for Type-I ROS $\cdot \mathrm{OH}$ characterization in the presence of TTCPy-1, TTCPy-2, TTCPy-3 or TTCPy-4 (0.5 mM in $\left.\mathrm{H}_{2} \mathrm{O}\right)$ before and after white light irradiation $\left(100 \mathrm{~mW} \mathrm{~cm}^{-2}\right)$.

higher than the initial intensity after 2-min white light irradiation. In addition, all these AIE-PSs showed much better overall ROS 
generation capability compared with commercially available Rose Bengal (RB) and Chlorine 6 (Ce6), the latter two are both very popular and reputable PSs.

We systematically investigated the species of ROS to have an in-depth understanding of the mechanism of ROS generated by these AIE-PSs. Initially, 9,10-anthracenediyl-bis(methylene) dimalonic acid (ABDA), a common used indicator was utilized for the detection of ${ }^{1} \mathrm{O}_{2}$ generation by these AIE-PSs. The absorption signal of ABDA treated with TTCPy-1, TTCPy-2, TTCPy-3 and TTCPy-4 respectively decreased about $4.19 \%$, $13.11 \%, 3.36 \%$ and $4.78 \%$, which was obviously lower than that treated with the commercial RB (33.22\%), implying their insufficient ${ }^{1} \mathrm{O}_{2}$ generation capacity (Figure 3B). The $\cdot \mathrm{OH}$ generation efficiency of these AIE-PSs was determined by comparing with $\mathrm{RB}$ and $\mathrm{Ce} 6$ under the same circumstances by using hydroxyphenyl fluorescein (HPF) as indicator. As illustrated in Figure $3 \mathrm{C}$, the fluorescence intensity of HPF enhanced more than 80 folds under continuous irradiation for 7 $\mathrm{min}$ in the presence of these AIE-PSs, further demonstrating that all of these AIE-PSs were capable of generating $\cdot \mathrm{OH}$ effectively through Type-I mechanism, while there was almost no emission for HPF alone under white light irradiation. Furthermore, a superoxide anion radical $\left(\mathrm{O}_{2}^{-\cdot}\right)$ probe, dihydrorhodamine 123 (DHR123), was used to estimate the $\mathrm{O}_{2}^{-\cdot}$ generation ability of these AIE-PSs. As displayed in Figure 3D, the PL intensity of DHR 123 in the TTCPy-2 and TTCPy-4 group after 7-min white light irradiation was over 48 -fold and 38 -fold of that before the irradiation respectively, suggesting significantly high $\mathrm{O}_{2}{ }^{-}$ generation efficiency of TTCPy-2 and TTCPy-4. Noteworthly, the $\mathrm{O}_{2}{ }^{-\cdot}$ generation capability of TTCPy-1 (31-fold) and TTCPy-3 (36-fold) was comparable with RB (34-fold), while significantly superior to $\mathrm{Ce} 6$ (2-fold). In addition, singlet oxygen sensor green (SOSG) was also utilized to detect Type-II $\mathrm{ROS}{ }^{1} \mathrm{O}_{2}$. There was almost no change of SOSG fluorescence in the presence of these AIE-PSs upon white light irradiation (Figure 3E), reflecting their infirm ${ }^{1} \mathrm{O}_{2}$ production efficiencies. The results from SOSG and ABDA indicators were consistent, which strongly confirmed the weak singlet oxygen generation ability and high free radical production capability from type I mechanism of these AIE-PSs.

To further confirm the $\cdot \mathrm{OH}$ and $\mathrm{O}_{2}^{-\cdot}$ production, 5,5dimethyl-1-pyrroline-N-oxide (DMPO) was used as the spin-trap agent to perform electron spin resonance (ESR) measurement. In the presences of DMPO and AIE-PSs, the resultant ESR spectrum displayed a typical four-line resonances with 1:2:2:1 intensity under white light irradiation (Figure 3F), which is the characteristic resonances for $\mathrm{DMPO} / \bullet \mathrm{OH}$ adduct. In addition, obvious six-line ESR signal was also identified, which origin from DMPO/O${ }_{2}^{-\bullet}$ adduct (Figure S21).

To profoundly study the mechanism of type I ROS generation, time-dependent density functional theory (TD-DFT) was carried out based on the B3LYP-D3/6-31G (d,p) level. As shown in Figure S22, the electron clouds of HOMOs of these AIE-PSs were mainly delocalized at the TPA moiety, while the LUMOs were primarily contributed by the orbitals of the cyano and pyridinium groups, suggesting the separation of HOMOs-LUMOs and their typical D-A characteristics. HOMOsLUMOs energy gaps of TTCPy-1, TTCPy-2, TTCPy-3 and TTCPy-4 were determined to be $2.17,2.14,2.17$ and $1.81 \mathrm{eV}$, respectively. The $\Delta E_{\text {st }}$ of TTCPy- $4(0.74 \mathrm{eV})$ with iodide $\left(\mathrm{I}^{-}\right)$as counterions was the smallest among these AIE-PSs $(1.22 \mathrm{eV}$, $1.20 \mathrm{eV}$ and $1.15 \mathrm{eV}$ for TTCPy-1, TTCPy-2 and TTCPy-3 respectively), implying that TTCPy-4 could generate free radicals more efficiently, which is in good accordance with the experimental results (Figure 3B). We further investigated electrochemical properties of TTCPy series by cyclic voltammetry using ferrocene $(\mathrm{Fc})$ as the external standard to help us understand why these AIE-PSs are prone to generating $\mathrm{O}_{2}^{-\cdot}$ and $\cdot \mathrm{OH}$ via the type I process. As depicted in Figure S23, the oxidation potentials of TTCPy-1, TTCPy-2, TTCPy- 3 and TTCPy-4 were located at $0.767 \mathrm{eV}, 0.709 \mathrm{eV}, 0.633 \mathrm{eV}$ and $0.234 \mathrm{eV}$, respectively, demonstrating the stronger electrondonating character of both $\mathrm{I}^{-}$and $\mathrm{Br}^{-}$ions, which endowed the excited TTCPy- 3 and TTCPy-4 PSs with easier access to electrons from electron-rich environment than TTCPy-1 and TTCPy-2, and then transfer an electron to molecular oxygen through type I mechanism. Therefore, the above theoretical and experimental results support our design concept that type I ROS can be generated by electron-rich anion- $\pi^{+}$AIE-PSs with enhanced ICT intensity and ISC process via acceptor engineering.

Since the nitrile-containing TTCPy series could enhance the binding affinity to the phospholipid membrane of bacterial cell walls through hydrogen bond interactions, ${ }^{[42]}$ leading to the restriction of the intramolecular motions of AIEgens and rapid enhancement of emission intensity, they are expected to realize the broad-spectrum imaging of $\mathrm{G}^{-}$and $\mathrm{G}^{+}$bacteria. We selected $S$. aureus and $E$. coli as representatives of $\mathrm{G}^{-}$and $\mathrm{G}^{+}$bacteria respectively. Confocal laser scanning microscopy (CLSM) showed that upon incubating $S$. aureus with $10 \mu \mathrm{M}$ AIE-PSs, the bright red fluorescence was clearly observed within 5 min with excellent contrast to the background (Figure 4A), indicating that these positive charged AIE-PSs have high binding affinity to peptidoglycan on the cell membrane of $\mathrm{G}^{+}$bacteria. Different from $\mathrm{G}^{+}$bacterial, the outer membrane in $\mathrm{G}^{-}$bacteria is a layer of the phospholipid membrane, which is an effective barrier to prevent the direct contact of extraneous invaders with the interbedded peptidoglycan network. Therefore, when incubated with previously reported AlEgens, there should be only weak fluorescence emission at the beginning. ${ }^{[40]}$ After 10 min

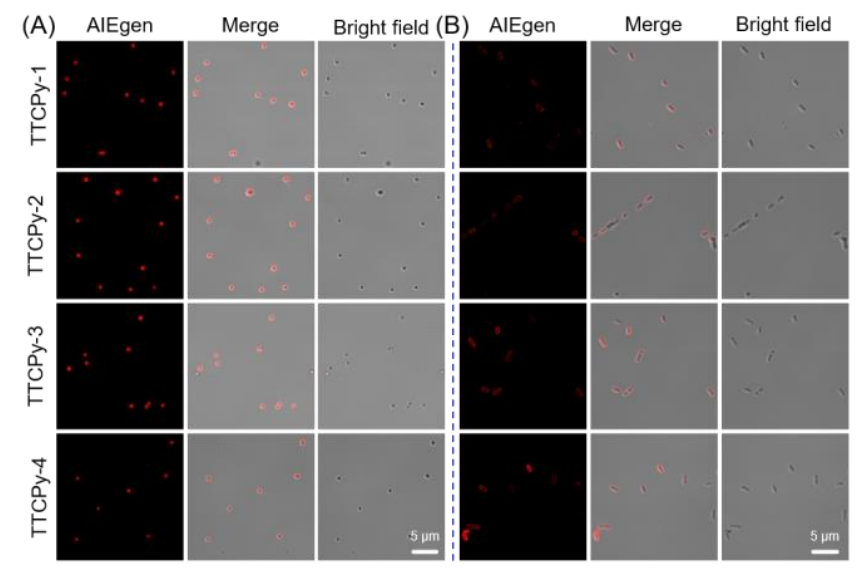

Figure 4. CLSM images of bacteria treated with AlEgens. (A) Bright-field and fluorescent images of MRSA incubated with $10 \mu \mathrm{M}$ of TTCPy-1, TTCPy-2, TTCPy-3 and TTCPy-4 for $5 \mathrm{~min}$. (B) Bright-field and fluorescent images of $E$. coli treated with $10 \mu \mathrm{M}$ of TTCPy-1 (10 min), TTCPy-2 (10 min), TTCPy-3 (15 $\mathrm{min}$ ) and TTCPy-4 (15 min) for different times. Ex $=488 \mathrm{~nm}, \mathrm{Em}=600-700 \mathrm{~nm}$. 

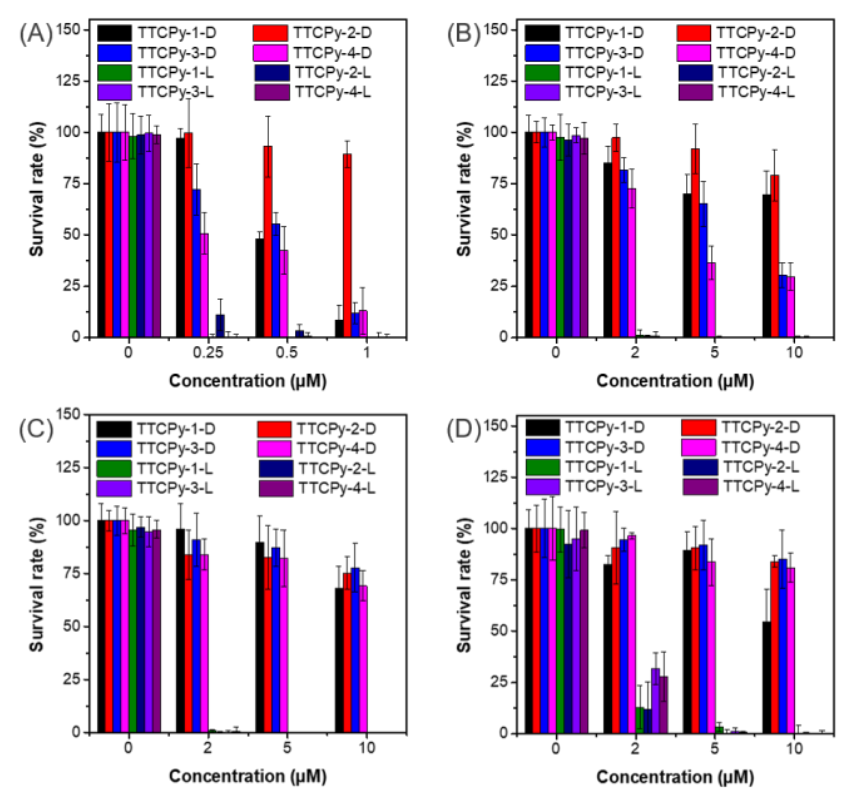

Figure 5. Survival rate of MRSA (A), E. coli (B), E. coli Top $10(\mathrm{C})$ and MDR $E$ coli (D) cultured on agar plate supplemented TTCPy-1, TTCPy-2, TTCPy-3 and TTCPy-4 at different concentrations in darkness (D) or upon white light irradiation (L) $\left(16 \mathrm{~mW} \mathrm{~cm}^{-2}\right)$ for $10 \mathrm{~min}(\mathrm{n}=3)$.

incubation of $E$. coli with TTCPy-1, TTCPy-2, TTCPy-3 and TTCPy-4, the fluorescent signals were clearly visualized in the bacteria. E. coli stained with TTCPy-3 and TTCPy-4 exhibited bright fluorescence with the prolongation of incubation time to 15 min. These results revealed that TTCPy series could achieve the broad-spectrum imaging of bacteria (Figure 4B).

Encouraged by the broad-spectrum imaging capability and high type I ROS $\left(\cdot \mathrm{OH}\right.$ and $\left.\mathrm{O}_{2}^{-}\right)$generation efficiency of these AIE-PSs, their antibacterial activity against MRSA, E. coli, E. coli Top 10 and MDR E. coli were investigated through the plate count method (Figure 5 and Figure S24-S27). In the absence of AIE-PSs, there was no obvious change in the survival rates of MRSA, E. coli, E. coli Top 10 and MDR E. coli in the dark or under white light irradiation. About $90 \%$ of MRSA incubated with TTCPy-1, TTCPy-3 and TTCPy-4 were killed at a concentration of $1 \mu \mathrm{M}$ under dark condition, while TTCPy-2 had a low MRSA killing efficacy under the same conditions. Compared with TTCPy-1 and TTCPy-2, both TTCPy-3 and TTCPy-4 had higher killing efficacies toward $E$. coli with a survival rate of $30 \%$ in the dark. The dark toxicity of these AIE-PSs to bacteria is a direct evidence of the interaction between nitrile-containing AlEgens and the bacterial cell walls. Upon white light irradiation, more than $99 \%$ of MRSA were killed at a low concentration of $0.25 \mu \mathrm{M}$ of TTCPy series except for TTCPy-2, suggesting a high photodynamic killing efficiency of TTCPy-1, TTCPy-3 and TTCPy-4 toward MRSA. This outcome can be reasonably attributed to the more efficient type I ROS generations of TTCPy-1, TTCPy-3 or TTCPy-4 than that of TTCPy-2. (Figure 3B). The survival rates of E. coli and E. coli Top 10 incubated with $2 \mu \mathrm{M}$ TTCPy-3 descended to less than $1 \%$ under white light irradiation $\left(16 \mathrm{~mW} \mathrm{~cm}^{-2}\right)$. When the concentration of AIE-PSs was increased to $5 \mu \mathrm{M}$, almost $100 \%$ of the bacteria were eradicated including the tricky MDR E. coli. The results indicate that these AIE-PSs have a profound and broad-spectrum PDT killing efficacy on MDR bacteria.
Cytotoxicity is a key parameter in the development of antibacterial PSs. An ideal antibacterial PS should not cause any damage to mammalian cells at the working concentrations. The biocompatibility of these AIE-PSs towards human umbilical vein endothelial cells (HUVECs) and healthy NIH 3 T3 cells were evaluated by MTT assay. As shown in Figure S29, there was negligible toxicity towards healthy HUVECs with the increasing concentration of AIE-PSs $(0.315,0.625,1.5,2.5$ and $5 \mu \mathrm{M})$ both in the dark and under the light irradiation, while the cell viability decreased obviously when treated with $10 \mu \mathrm{M}$ TTCPy-1 and TTCPy-4 under light irradiation. The same trend of cell viability were observed in healthy NIH3T3 cells incubated with these four AIE-PSs, indicating that TTCPy-2 and TTCPy-3 had a better performance in biocompatibility (Figure S30).

To investigate the antibacterial mechanism and gain more insights into the structure-function relationships of TTCPy series, we utilized FE-SEM and FEHR-TEM to visualize the morphological changes of the bacteria incubated with TTCPy-1, TTCPy-2, TTCPy-3 and TTCPy-4 in dark or under white light irradiation, selecting E. coli $\left(\mathrm{G}^{-}\right)$and MRSA $\left(\mathrm{G}^{+}\right)$as representative bacterials. E. coli in the AIE-PSs@Light groups showed obvious caves on the cell walls, while the morphology of bacteria in the dark barely changed (Figure 6A and 6B). The cell

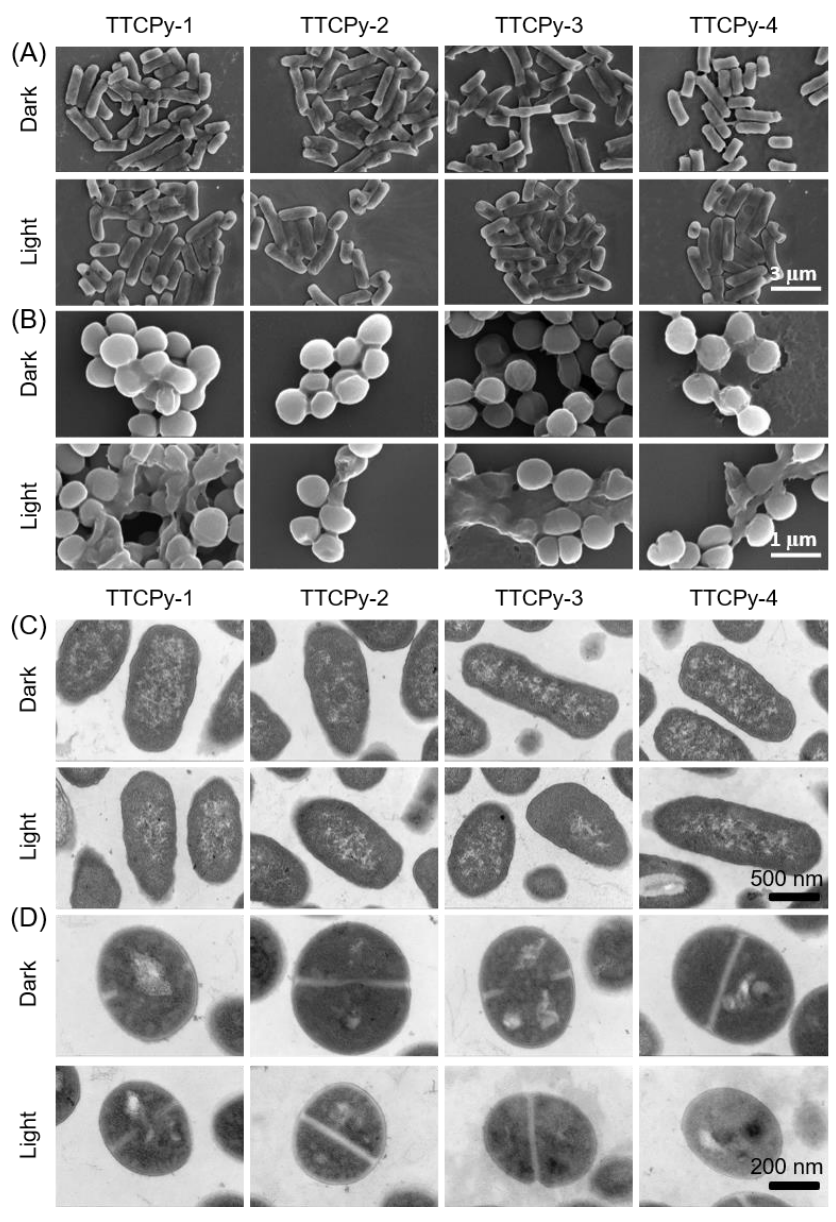

Figure 6. Visualizing TTCPy-1, TTCPy-2, TTCPy-3 and TTCPy-4 induced morphological changes of $E$. coli $(\mathrm{A})$ and MRSA (B) in darkness or upon white light irradiation (16 $\mathrm{mW} \mathrm{cm}^{-2}$ ) by FE-SEM. FEHR-TEM images of TTCPy-1, TTCPy-2, TTCPy-3 and TTCPy-4 induced morphological changes of E. coli (C) and MRSA (D) in darkness or upon white light irradiation $\left(16 \mathrm{~mW} \mathrm{~cm}^{-2}\right)$. 
A Control TTCPy-3+L Control TTCPy-3 TTCPy-3+L B
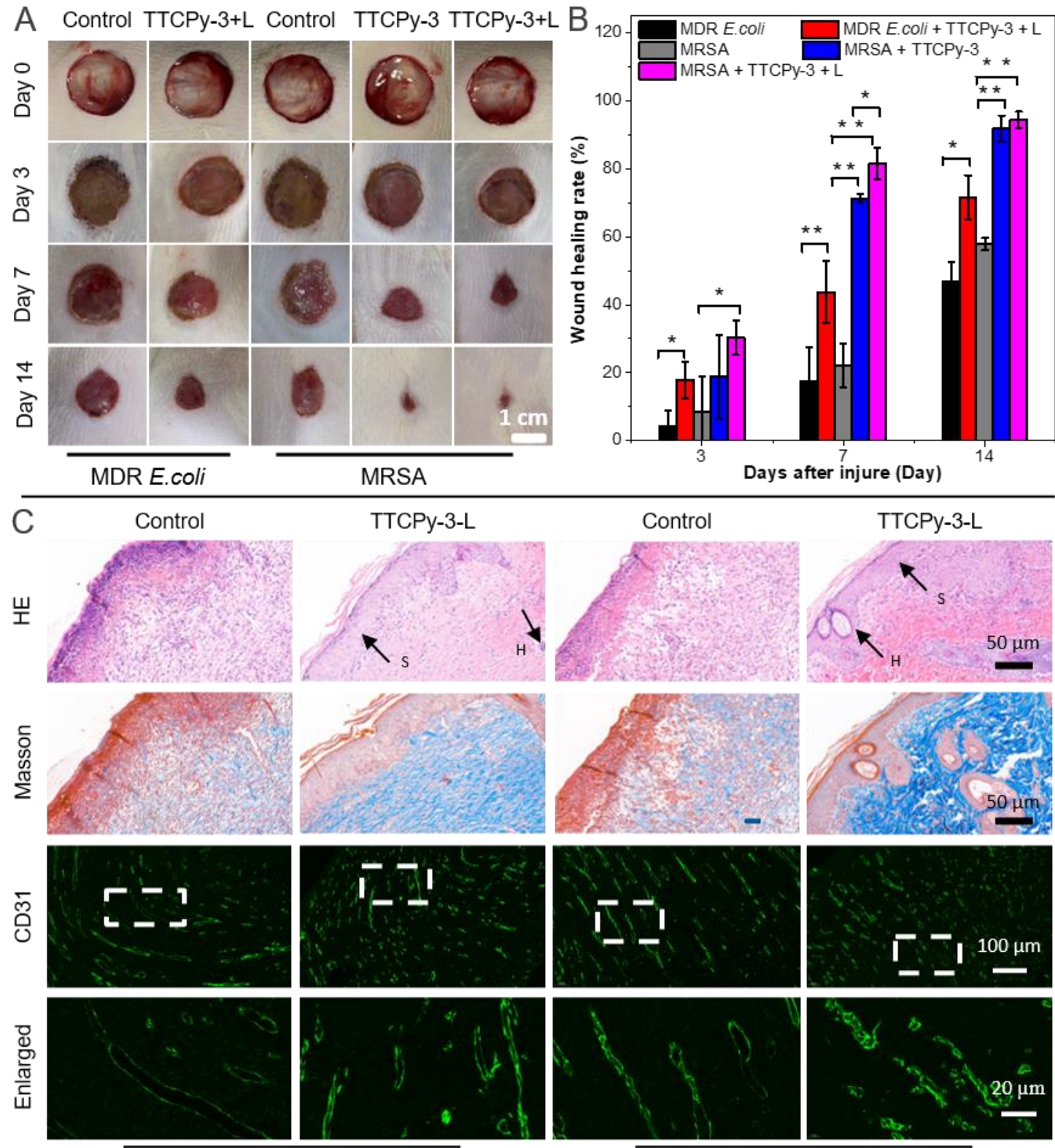

MDR E.coli

MRSA

Figure 7. In vivo evaluation of TTCPy-3 in treatment of MDR E. coli- and MRSA-infected wounds on rats. (A) Photographs of infected wounds treated by TTCPy- 3 or TTCPy-3 plus white-light irradiation after injury for different time periods. (B) The proportion of the MDR E. coli- and MRSA-infected wound area on day 3, day 7 and day 14 after the injury $(\mathrm{n}=3)$. The data show significant statistical differences between MDR E. coli- and MRSA-infected groups and MDR E. coli- and MRSAinfected wounds treated by TTCPy-3 with or without light irradiation, MRSA-infected wounds treated by TTCPy-3 with or without light irradiation (16 mW $\mathrm{cm}^{-2}$ ) $\left(0.001<{ }^{*} \mathrm{P}<0.05,{ }^{* *} \mathrm{P}<0.001\right)$. (C) HE, Masson and CD 31 staining of the sectioned tissues of wounds on rats. MDR E. coli-, MRSA-infected wounds were treated with or without TTCPy-3 plus white-light irradiation. The tissues were resected on day 14 after injury. The letters in the images indicate specific cell types and structures in the histological sections. $\mathrm{H}$, hair follicle; $\mathrm{S}$, squamous epithelial cell.

walls of MRSA collapsed dramatically in the presence of AIEPSs with white light irradiation $\left(16 \mathrm{~mW} \mathrm{~cm}^{-2}\right)$. Additionally, FEHR-TEM analysis was performed to visualize the morphological features of $E$. coli and MRSA before and after PDT treatment. It was observed that the edge of $E$. coli cell wall became blurred under AIE-PSs and light irradiation compared with the control groups (Figure $6 \mathrm{C}$ ). The outer surfaces of MRSA bacteria incubated with the AIE-PSs were destroyed more obviously in the presence of light irradiation. The antibacterial effects of these AIE-PSs are mainly originated from the inactivation of biomacromolecules by free radicals generated from type I mechanism upon white light irradiation.

Based on the in vitro antibacterial results of these AIE-PSs, TTCPy-3 was selected to further evaluate the antibacterial effect in vivo. Animal models with MRSA and MDR E. coli-infected wounds on the dorsal skin of Wistar rats were established respectively. For the rats infected with MDR E. coli, the rats were randomly divided into two groups: 1) treated with phosphate-buffered saline (PBS) only (control group); 2) TTCPy$3 @$ white-light (PDT group). Considering the excellent dark toxicity of TTCPy- 3 to $\mathrm{G}^{+}$bacteria, the rats infected with MRSA 
were randomly assigned to three groups for the following treatments: PBS only (control group), TTCPy-3 only (dark group) and TTCPy-3@white-light (PDT group) (Figure 7A).

In order to clearly observe the healing process, the macroscopic appearance of the wounds were captured at different time points. At day 3 postinfection, there was no significant difference in all the wounds. At day 7 , the sizes of the MDR E. coli-infected wounds treated with TTCPy-3@white-light irradiation (16 mW cm${ }^{-2}, 30 \mathrm{~min}$ ) was apparently smaller than that of the control group, while the sizes of MRSA-infected wounds treated with TTCPy-3 only (dark group) and TTCPy-

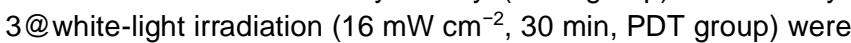
significantly smaller in comparison to the control group treated with PBS only. At day 14, the MDR E. coli-infected wounds treated with TTCPy-3@white-light irradiation were much smaller and cleaner than the control group, and the wound healing rate was over $70 \%$. Meanwhile, the MRSA-infected wounds treated with TTCPy-3 only and TTCPy-3@white light had almost recovered with a wound healing rate up to $91.7 \%$ and $94.4 \%$, respectively (Figure 7B). Moreover, hematoxylin and eosin $(\mathrm{H} \& \mathrm{E})$ and masson staining of the sectioned tissues of the wounds on rats were utilized to evaluate the wound-healing efficacy. ${ }^{[52]}$ As clearly shown in Figure $7 \mathrm{C}$, at day 14, intact and thick epidermis appeared in the neonatal skin of the PDT groups. Moreover, hair follicles and squamous epithelial cells can be clearly observed on the sectioned tissues of the wounds from the same group, strongly indicating the excellent wound healing efficacy of TTCPy-3 in the presence of light irradiation. Similarly, the collagen fibers which dyed blue could be visualized clearly in the neonatal skin after Masson's trichrome staining, demonstrating a much more prominent antibacterial efficiency in the PDT groups. Finally, CD31 staining was performed and the images indicated that more blood vessels were observed in the neonatal structure of PDT groups. Therefore, the in vivo results strongly demonstrated that TTCPy-3 has a supremely prominent light-enhanced antimicrobial effect toward MDR bacteria and can dramatically promote the wound-healing process, which is highly consistent with antibacterial tests in vitro.

\section{Conclusion}

In summary, we develpoed a series of NIR anion- $\pi^{+}$AIE-PSs (TTCPy-1, TTCPy-2, TTCPy-3 and TTCPy-4) with efficient ROS generation via the type I mechanism, which can be used at very low concentrations to effectively kill drug-resistant bacteria strains. Theoretical calculations and experimental results suggested that strong $\mathrm{D}-\mathrm{A}$ effect and sufficient small $\Delta E_{\mathrm{ST}}$ play dominate roles in the ISC process, which is in favor of producing destructive type I ROS. Thanks to the broad-spectrum bacterial binding ability and efficient Type I ROS generation capability, TTCPy series exhibited excellent photodynamic antibacterial activity in destroying MRSA, E. coli, E. coli Top 10 and MDR E. coli at low concentration $(0.25 \mu \mathrm{M}$ for MRSA, $2 \mu \mathrm{M}$ for E.coli and E.coli Top 10, $5 \mu \mathrm{M}$ for MDR E.coli) and low light dose (16 mW $\mathrm{cm}^{-2}$ ). Benefiting from its excellent biocompatibility and bright emission in the NIR region, TTCPy-3 has been utilized for photodynamic inactivation of MRSA and MDR E.coli in vivo, leading to successfully suppression of the bacterial infections in wounds. Hence, our study not only provides a new strategy for the design of type I AIE photosensitizers as next-generation advanced antimicrobial agents, but also offers new insights for preclinical research and clinical applications to combat bacteria resistance.

\section{Acknowledgements}

This work was supported by the Natural Science Foundation of China $(22007067,22005195,21801169)$, the China Postdoctoral Science Foundation Grant (2020M672768), the Science and Technology Foundation of Shenzhen City (JCYJ20190808153415062, JCYJ20190808121417291, RCYX20200714114525101), and the Natural Science Foundation for Distinguished Young Scholars of Guangdong Province (2020B1515020011). The authors also acknowledge the Instrumental Analysis Center of Shenzhen University. The Hela, A549 and 4T1 cell lines were purchased from Chinese Academy of Science Cell Bank for Type Culture Collection.

Keywords: aggregation-induced emission $\cdot$ multidrug resistance bacteria $\cdot$ photosensitizers $\cdot$ type I ROS $\cdot$ photodynamic therapy

[1] A. Zlitni, G. Gowrishankar, I. Steinberg, T. Haywood, S. Sam Gambhir, Nat. Commun. 2020, 11, 1250.

[2] B. Spellberg, R. Guidos, D. Gilbert, J. Bradley, H. W. Boucher, W. M. Scheld, J. G. Bartlett, J. Edwards, Jr, t. I. D. S. o. America, Clin. Infect. Dis. 2008, 46, 155-164.

[3] S. Nolivos, J. Cayron, A. Dedieu, A. Page, F. Delolme, C. Lesterlin, Science 2019, 364, 778-782.

[4] P. Kardas, S. Devine, A. Golembesky, C. Roberts, Int. J. Antimicrob. Agents 2005, 26, 106-113.

[5] A. J. Alanis, Arch. Med. Res. 2005, 36, 697-705

[6] T. T. Yoshikawa, J. Am. Geriatr. Soc. 2002, 50, 226-229.

[7] E. D. Brown, G. D. Wright, Nature 2016, 529, 336-343.

[8] M. Baym, L. K. Stone, R. Kishony, Science 2016, 351, aad3292.

[9] WHO. Artemisinin resistance and artemisinin-based combination therapy efficacy https:// www.who.int/docs/defaultsource/documents/publications/gmp/who-cds-gmp-2019eng.pdf?ua=1 (World Health Organization, 2019).

[10] J. P. Celli, B. Q. Spring, I. Rizvi, C. L. Evans, K. S. Samkoe, S. Verma, B. W. Pogue, T. Hasan, Chem. Rev. 2010, 110, 2795-2838.

[11] X. Li, S. Lee, J. Yoon, Chem. Soc. Rev. 2018, 47, 1174-1188.

[12] A. Almeida, M. A. Faustino, J. P. Tomé, Future Med. Chem. 2015, 7, 1221-1224.

[13] H. Yuan, H. Chong, B. Wang, C. Zhu, L. Liu, Q. Yang, F. Lv, S. Wang, J. Am. Chem. Soc. 2012, 134, 13184-13187.

[14] T. J. Dougherty, C. J. Gomer, B. W. Henderson, G. Jori, D. Kessel, M. Korbelik, J. Moan, Q. Peng, JNCI, J. Natl. Cancer Inst. 1998, 90, 889905.

[15] W. Wu, D. Mao, F. Hu, S. Xu, C. Chen, C.-J. Zhang, X. Cheng, Y. Yuan, D. Ding, D. Kong, B. Liu, Adv. Mater. 2017, 29, 1700548.

[16] W. Wu, D. Mao, X. Cai, Y. Duan, F. Hu, D. Kong, B. Liu, Chem. Mater. 2018, 30, 3867-3873.

[17] J. F. Lovell, T. W. B. Liu, J. Chen, G. Zheng, Chem. Rev. 2010, 110, 2839-2857.

[18] K. Plaetzer, B. Krammer, J. Berlanda, F. Berr, T. Kiesslich, Lasers Med. Sci. 2009, 24, 259-268.

[19] S. Pervaiz, M. Olivo, Clin. Exp. Pharmacol. Physiol. 2006, 33, 551-556.

[20] S. Morley, J. Griffiths, G. Philips, H. Moseley, C. O'Grady, K. Mellish, C. L. Lankester, B. Faris, R. J. Young, S. B. Brown, L. E. Rhodes, Br. J. Dermatol. 2013, 168, 617-624.

[21] J. P. Tardivo, F. Adami, J. A. Correa, M. A. S. Pinhal, M. S. Baptista, Photodiagn. Photodyn. Ther. 2014, 11, 342-350.

[22] X. Lei, B. Liu, Z. Huang, J. Wu, Arch. Dermatol. Res. 2015, 307, 49-55. 
[23] A. Oniszczuk, K. A. Wojtunik-Kulesza, T. Oniszczuk, K. Kasprzak, Biomed. Pharmacother. 2016, 83, 912-929.

[24] A. Naranjo, A. Arboleda, J. D. Martinez, H. Durkee, M. C. Aguilar, N. Relhan, N. Nikpoor, A. Galor, S. R. Dubovy, R. Leblanc, H. W. Flynn Jr, D. Miller, J.-M. Parel, G. Amescua, Am. J. Ophthalmol. 2019, 208, 387396.

[25] L. E. Bennett, K. P. Ghiggino, R. W. Henderson, J. Photochem. Photobiol., B 1989, 3, 81-89.

[26] J. Luo, Z. Xie, J. W. Y. Lam, L. Cheng, H. Chen, C. Qiu, H. S. Kwok, X. Zhan, Y. Liu, D. Zhu, B. Z. Tang, Chem. Commun. 2001, 1740-1741.

[27] J. Mei, N. L. C. Leung, R. T. K. Kwok, J. W. Y. Lam, B. Z. Tang, Chem. Rev. 2015, 115, 11718-11940.

[28] G. Chen, W. Li, T. Zhou, Q. Peng, D. Zhai, H. Li, W. Z. Yuan, Y. Zhang B. Z. Tang, Adv. Mater. 2015, 27, 4496-4501.

[29] Y. Li, Z. Zhao, J. Zhang, R. T. K. Kwok, S. Xie, R. Tang, Y. Jia, J. Yang, L. Wang, J. W. Y. Lam, W. Zheng, X. Jiang, B. Z. Tang, Adv. Funct. Mater. 2018, 28, 1804632.

[30] S. Chen, Q. Chen, Q. Li, J. An, P. Sun, J. Ma, H. Gao, Chem. Mater. 2018, 30, 1782-1790.

[31] Q. Li, Y. Li, T. Min, J. Gong, L. Du, D. L. Phillips, J. Liu, J. W. Y. Lam, H. H. Y. Sung, I. D. Williams, R. T. K. Kwok, C. L. Ho, K. Li, J. Wang, B. Z. Tang, Angew. Chem. Int. Ed. 2020, 59, 9470-9477.

[32] F. Hu, G. Qi, Kenry, D. Mao, S. Zhou, M. Wu, W. Wu, B. Liu, Angew. Chem. Int. Ed. 2020, 59, 9288-9292.

[33] G. Qi, F. Hu, Kenry, K. C. Chong, M. Wu, Y. H. Gan, B. Liu, Adv. Funct Mater. 2020, 30, 2001338.

[34] J.-S. Ni, T. Min, Y. Li, M. Zha, P. Zhang, C. L. Ho, K. Li, Angew. Chem Int. Ed. 2020, 59, 10179-10185.

[35] T. Zhou, R. Hu, L. Wang, Y. Qiu, G. Zhang, Q. Deng, H. Zhang, P. Yin, B. Situ, C. Zhan, A. Qin, B. Z. Tang, Angew. Chem. Int. Ed. 2020, 59 9952-9956.

[36] S. Gao, X. Yan, G. Xie, M. Zhu, X. Ju, P. J. Stang, Y. Tian, Z. Niu, Proc Natl. Acad. Sci. U.S.A. 2019, 116, 23437-23443.

[37] X. He, Y. Yang, Y. Guo, S. Lu, Y. Du, J.-J. Li, X. Zhang, N. L. C. Leung, Z. Zhao, G. Niu, S. Yang, Z. Weng, R. T. K. Kwok, J. W. Y. Lam, G. Xie B. Z. Tang, J. Am. Chem. Soc. 2020, 142, 3959-3969.

[38] R. Hu, Q. Deng, Q. Tang, R. Zhang, L. Wang, B. Situ, C. Gui, Z. Wang, B. Z. Tang, Biomaterials 2021, 271, 120725.

[39] M. Kang, C. Zhou, S. Wu, B. Yu, Z. Zhang, N. Song, M. M. S. Lee, W. Xu, F.-J. Xu, D. Wang, L. Wang, B. Z. Tang, J. Am. Chem. Soc. 2019 , 141, 16781-16789.

[40] H. Bai, W. He, J. H. C. Chau, Z. Zheng, R. T. K. Kwok, J. W. Y. Lam, B. Z. Tang, Biomaterials 2021, 268, 120598.

[41] F. F. Fleming, L. Yao, P. C. Ravikumar, L. Funk, B. C. Shook, J. Med. Chem. 2010, 53, 7902-7917.

[42] Y. Wang, Y. Du, N. Huang, Future Med. Chem. 2018, 10, 2713-2728.

[43] Q. Wan, R. Zhang, Z. Zhuang, Y. Li, Y. Huang, Z. Wang, W. Zhang, J. Hou, B. Z. Tang, Adv. Funct. Mater. 2020, 30, 2002057.

[44] X. Zhao, Y. Dai, F. Ma, S. Misal, K. Hasrat, H. Zhu, Z. Qi, Chem. Eng. J. 2021, 410, 128133

[45] C. M. Marian, WIREs Comput. Mol. Sci. 2012, 2, 187-203.

[46] J. Zhao, W. Wu, J. Sun, S. Guo, Chem. Soc. Rev. 2013, 42, 5323-5351.

[47] B. L. Schottel, H. T. Chifotides, K. R. Dunbar, Chem. Soc. Rev. 2008, 37, 68-83.

[48] A. Frontera, P. Gamez, M. Mascal, T. J. Mooibroek, J. Reedijk, Angew. Chem. Int. Ed. 2011, 50, 9564-9583.

[49] J. Wang, X. Gu, H. Ma, Q. Peng, X. Huang, X. Zheng, S. H. P. Sung, G. Shan, J. W. Y. Lam, Z. Shuai, B. Z. Tang, Nat. Commun. 2018, 9, 2963.

[50] S. Xu, W. Wu, X. Cai, C.-J. Zhang, Y. Yuan, J. Liang, G. Feng, P. Manghnani, B. Liu, Chem. Commun. 2017, 53, 8727-8730.

[51] L. Yang, X. Wang, G. Zhang, X. Chen, G. Zhang, J. Jiang, Nanoscale 2016, 8, 17422-17426.

[52] Y. Li, S. Wang, R. Huang, Z. Huang, B. Hu, W. Zheng, G. Yang, X. Jiang, Biomacromolecules 2015, 16, 780-789. 


\section{Entry for the Table of Contents}

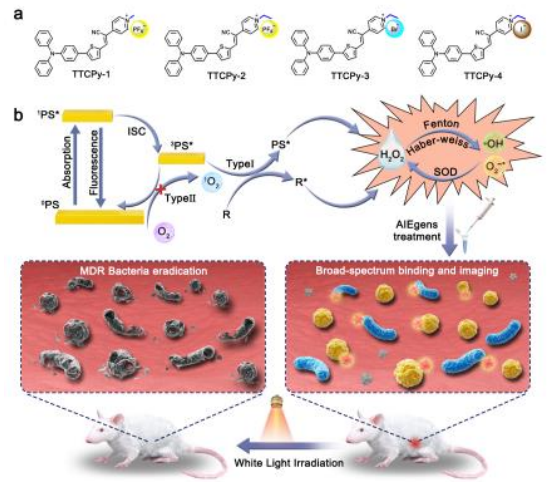

A series of near-infrared anion- $\Pi^{+}$photosensitizers featuring aggregation-induced emission (AIE-PSs) were developed for broadspectrum multidrug-resistance bacteria eradication. These electron-rich anion- $\pi^{+}$AIE-PSs with strong intramolecular charge transfer character showed boosted type I reactive oxygen species (ROS) generation capability, achieving a high photodynamic killing efficacy toward both Methicillin-resistant Staphylococcus aureus (MRSA) and multidrug-resistance Escherichia coli (MDR E. coli) under a low dose white light irradiation $\left(16 \mathrm{~mW} \mathrm{~cm}^{-2}\right)$ in vitro and in vivo. 